INPLASY

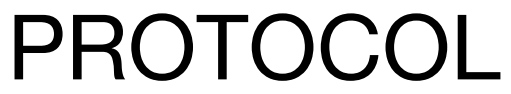

To cite: Qiao et al. The efficacy of adjunctive therapy using Huanshao Dan (HSD) in patients with dementia: $A$ protocol for systematic review and meta-analysis of randomized controlled trials. Inplasy protocol 2020120001. doi:

10.37766/inplasy2020.12.0001

Received: 14 December 2020

Published: 15 December 2020

Corresponding author:

Yiqiang Xie

mrxieyiqiang@163.com

Author Affiliation:

The Seventh People's Hospital

Support: ZDYF2019210.

Review Stage at time of this submission: Preliminary searches.

Conflicts of interest: None.

\section{The efficacy of adjunctive therapy using Huanshao Dan (HSD) in patients with dementia: A protocol for systematic review and meta-analysis of randomized controlled trials}

Qiao, LJ1; Xie, YQ2; Dong, XJ3; Hu, HM4; Ma, TP5; Oliver, BG6; Chen, $\mathrm{H}^{7}$.

Review question / Objective: The efficacy of adjunctive therapy using Huanshao Dan (HSD) in patients with dementia. Condition being studied: Dementia is one of the most common neurodegenerative disorders in the aging population. Albeit the ongoing effort of new drug development, there is no cure, neither effective treatment to stop the progression. Herbal concoction Huanshao Dan (HSD) has been used to treat memory decline in China for centuries, including in patients with dementia in recent years. However, there is no systematic review and meta-analysis to examine its efficacy in this setting.

INPLASY registration number: This protocol was registered with the International Platform of Registered Systematic Review and Meta-Analysis Protocols (INPLASY) on 15 December 2020 and was last updated on 15 December 2020 (registration number INPLASY2020120082).

\section{INTRODUCTION}

Review question / Objective: The efficacy of adjunctive therapy using Huanshao Dan (HSD) in patients with dementia.
Condition being studied: Dementia is one of the most common neurodegenerative disorders in the aging population. Albeit the ongoing effort of new drug development, there is no cure, neither effective treatment to stop the progression. Herbal concoction Huanshao Dan (HSD) has been used to 
treat memory decline in China for centuries, including in patients with dementia in recent years. However, there is no systematic review and meta-analysis to examine its efficacy in this setting.

\section{METHODS}

Search strategy: The search terms will combine all the possible keywords for the research topic, which include, ("dementia", OR "Alzheimer's disease" OR "dementia with Lewy bodies" OR "vascular dementia") AND ("Huanshao Capsules" OR "Huanshao Dan" OR "Huanshaodan") AND ("Randomised controlled trial" OR "RCT" OR "clinical study" OR "clinical trials"). Both Chinese and English terms will be used for different databases. Articles will be retrieved from the inception to December 2021.

Participant or population: The study population will be patients with a confirmed diagnosis of dementia.

Intervention: If the control group did not receive any treatment, the interventions in the study group can include any conventional treatment with and without the combination of HSD. If the control group receives conventional treatment, the intervention in the study group needs to be a combination of this conventional therapy and HSD. In both settings, a study group with HSD mono-therapy can be included. HSD mono-therapy and combined therapy. Any dose of HSD is included.

Comparator: Any type of control group. The control group used placebo control or no Treatment or conventional medication such as Donepezil, rivastigmine, galantamine.

Study designs to be included: We will include any randomized controlled trials that discusses the efficacy of HSD in patients with dementia.

Eligibility criteria: Participants: The study population will be patients with a confirmed diagnosis of dementia according to the international standard . They should have any major organ dysfunction (including the heart, lung, kidney, and liver), illness (e.g. cancer), and pregnancy. There is no restriction on the sex, race, ethnicity, birthplace, residential address, and age of the participants in the study. Interventions: If the control group did not receive any treatment, the interventions in the study group can include any conventional treatment with and without the combination of HSD. If the control group receives conventional treatment, the intervention in the study group needs to be a combination of this conventional therapy and HSD. In both settings, a study group with HSD mono-therapy can be included. HSD mono-therapy and combined therapy. Any dose of HSD is included. Control: Any type of control group. The control group used placebo control or no Treatment or conventional medication such as Donepezil, rivastigmine, galantamine. Outcome: The primary outcomes are the results of three questionnaires, including mini-mental state examination (MMSE), Hasegawa's DementiaScale (HDS-R), and activities of daily living (ADL ). Type: We will include any randomized controlled trials that discusses the efficacy of HSD in patients with dementia.

Information sources: We will perform a systemic literature search in 3 English databases (PubMed, Cochrane Central Register of Controlled Trials (CENTRAL), Web of Science) and 4 Chinese databases (VIP information database, Chinese Biomedical Literature Database, Chinese National Knowledge Infrastructure (CNKI), and Wanfang Database).

Main outcome(s): The primary outcomes are the results of three questionnaires, including mini-mental state examination (MMSE), Hasegawa's DementiaScale (HDS$R)$, and activities of daily living (ADL).

Quality assessment / Risk of bias analysis: The Cochrane Handbook criteria for judging the RoB with the"Risk of bias" assessment tool will be used to assess the risk of literature bias. The quality of the methodology will be assessed by two independent reviewers using RevMan 5.3. 
The following seven domains will be evaluated, including blinding of the participants and investigators, random sequence generation, the blindness of outcome assessments, allocation concealment selective outcome reporting, incomplete outcome data, and other biases. As a result, the quality of studies will be judged as unclear, low, or high bias.

Strategy of data synthesis: We will use RevMan 5.3 statistical software for metaanalysis. The relative risk (RR) will be used as the treatment effect value; the mean difference (MD) will be used as the treatment effect value and to calculate its $95 \%$ confidence interval $(\mathrm{Cl}) .12$ will be used to analyse the heterogeneity. When $12<$ $50 \%$, the fixed effect model is used for consolidation analysis; if $12 \geq 50 \%$, the heterogeneity among the studies is considered significant, and the random effect model is selected for data consolidation analysis. When the heterogeneity is large, the sensitivity analysis will be performed by removing individual literature one by one, and the impact of a single study on the combined effect will be analysed.

Subgroup analysis: If the studies show significant heterogeneity. subgroup analysis will be performed to explore the source of heterogeneity.

Sensibility analysis: Furthermore if necessary, a sensitivity analysis will be performed.

Country(ies) involved: China.

Keywords: protocol, adjunctive treatment, dementia, RCTs, Huanshao Dan (HSD).

Contributions of each author:

Author 1 - Lijun Qiao.

Author 2 - Yiqiang Xie.

Author 3 - Xiujuan Dong.

Author 4 - Hongming Hu.

Author 5 - Tianpeng Ma.

Author 6 - Brian G Oliver. 